\title{
Laser Ablation Inductively Coupled Mass Spectrometry for in situ Trace Element and Isotope Analysis
}

\author{
R. D. Ash*
}

* Department of Geology, University of Maryland, College Park, MD 20742 USA

Although laser ablation inductively coupled mass spectrometry (LA-ICPMS) has been used for over a decade usage has exploded in the past 4-5 years. This is the result of improvements in mass spectrometry and in off-the-shelf laser ablation systems. The technique allows the in situ measurement of trace element abundances down to ppb levels [e.g.1] and isotopic ratios with sub per mil precision [2]. It has been applied widely in the material science and geological communities and increasingly applications are being found in archeological [3], environmental and, more recently, in the forensic and biological fields [4].

The measurement of trace element abundances has many uses in the geological sciences; e.g. thermometry, barometry, material provenance, chronology [5]. Whilst there are several techniques for making such measurements, including ion microprobe and proton probe, these methods have tended to have certain logistical drawbacks, not least time, cost and instrument accessibility. With the increasing availability of LA-ICPMS systems this method is rapidly becoming the instrument of choice in the geological community for making measurements of trace element abundances.

The LA-ICPMS system typically consists of a sample cell in which samples and standards are mounted, a laser (typically in the UV part of the spectrum) which fires though a window into the cell whence particles are ablated into a stream of He for transportation into ionizing plasma, thence into the mass spectrometer. The mass spectrometer may be a quadropole or, increasingly, a magnetic sector instrument with one or more collectors for measurement of mass/charge of the ions produced.

Why use LA-ICPMS? As well as increasing availability of instrumentation there are sound technical reasons for the burgeoning usage of LA-ICPMS. Just about any solid material can be analysed, as long as there is coupling between the laser light and the sample, that suitable external standard material is available and that an appropriate element is known for use as an internal standard. Compared with secondary ion mass spectrometry (SIMS/ion probe) the method is forgiving in terms of matrix matching, although egregious differences in matrices are to be avoided (e.g. metals vs. silicates vs. sulphides vs. organics). The recent development of carbonate, sulphide and phosphate standards by the USGS complement the already well characterised silicate and metal standards that are widely used, although organic standards remain problematic. The laser is able to ablate material from relatively rough and non-conducting surfaces so sample preparation is quick and easy. Laser ablation also enables the rapid measurement of trace element concentrations, with the analysis for multiple elements typically taking a few minutes, including time for the flushing out of the ablation cell. In complex samples data processing may be time consuming but increasingly automated data management for simple systems is widespread.

As well as simple measurement of concentration laser ablation allows relatively simple measurement of elemental or isotopic profiles. If the ablation rate, typically $c a$. $100 \mathrm{~nm}$ per 
laser shot, is known then there is a simple correlation between time and depth, until attenuation and potential fractionation occur with high depth to diameter aspect ratios. Alternatively the sample cell may be moved beneath the laser to traverse grain boundaries or along diffusion profiles.

Disadvantages. Just as with any technique there are disadvantages with LA-ICPMS. As with most mass spectrometric techniques elemental and molecular interferences are potentially problematic. These may be mitigated by choice of appropriate isotopes for measurement, or by increasing mass resolution with a magnetic sector instrument or selecting an appropriate gas in collision cell quadropole instruments. Although there are many advantages to laser ablation it remains a destructive technique. Spot size requirements are directly related to concentration of elements of interest. Even using high sensitivity magnetic sector instruments analysis of elements present at the low ppm level require ablation pits of 30-40 $\mu \mathrm{m}$ diameter, but if the concentrations of elements are at the sub$10 \mathrm{ppb}$ level then spot sizes in the $200-250 \mu \mathrm{m}$ size would be appropriate [1]. In many applications this is not of great import but for applications where sample destruction is not an option, for example with many unique archeological and cultural objects this may be prohibitive.

The future of LA-ICPMS. Experiments have shown that shorter laser wavelengths generally produce a smaller and more uniform particle size, which is advantageous to uniform behaviour in the plasma for vapourisation and ionisation. Early ablation was typically carried out using 266nm light (quadrupled Nd:YAG laser), whereas now 213nm and 193nm wavelengths (from NdYAG and ArF lasers) are widely available and give considerable stability and reproducibility advantages. These shorter wavelengths also enable the ablation of a wider variety of materials that have less interaction with longer wavelength light. However research has also shown that the use of very short duration light pulses (femtosecond lasers) allow stoichiometric ablation with longer wavelength light.

Ongoing mass spectrometric developments include increasing the dynamic range of detectors, to both higher sensitivity for lower concentration measurements, and also methods for the measurement of higher concentration elements without detector saturation.

Other areas of active development is in the increasing use of multicollector ICPMS for in situ measurement of non-conventional stable isotope systems and for rapid determination of geological ages.

[1] R. Arevalo Jr. and W.F. McDonough, Earth Planet. Sci. Lett. 272, (2008) 656.

[2] E.D. Young, R.D. Ash, A. Galy, N.S. Belshaw, Geochim. Cosmochim. Acta 66, (2005) 683.

[3] R.J. Speakman and R.J. Neff (eds.) Laser Ablation ICP-MS in Archaeological Research. University of New Mexico Press (2005).

[4] A.M. Ghazi in Introduction to Environmental Forensics $2^{\text {nd }}$ Murphy \& Morrison (eds.) (2007) 637.

[5] J.I. Simon et al., (2005) Earth Planet. Sci. Lett. 238, 272. 